Plasma-wall interaction on the divertor tiles of JET ITER-like wall from the viewpoint of micro/nanoscopic observations

\title{
JET Contributors
}

2018-11

JET Contributors , Tokitani , M , Miyamoto, M , Masuzaki , S , Sakamoto , R, Oya , Y , Hatano , Y, Otsuka , T , Oyaidzu, M , Kurotaki , H, Suzuki , T, Hamaguchi , D , Isobe , K, Asakura, N, Widdowson , A, Heinola , K, Rubel , M \& Ahlgren , T 2018 , ' Plasma-wall interaction on the divertor tiles of JET ITER-like wall from the viewpoint of micro/nanoscopic observations ' , Fusion Engineering and Design , vol. 136 , pp. 199-204 . https://doi.org/10.1016/j.fusengdes.2018.01

http://hdl.handle.net/10138/326291

https://doi.org/10.1016/j.fusengdes.2018.01.051

cc_by_nc_nd

acceptedVersion

Downloaded from Helda, University of Helsinki institutional repository.

This is an electronic reprint of the original article.

This reprint may differ from the original in pagination and typographic detail.

Please cite the original version. 


\title{
Plasma-Wall Interaction on the Divertor Tiles of JET ITER-Like Wall from the Viewpoint of Micro/Nanoscopic Observations
}

\author{
M. Tokitani ${ }^{\text {a* }}$, M. Miyamoto ${ }^{\text {b }}$, S. Masuzaki ${ }^{\text {a }}$, R. Sakamoto ${ }^{\text {a }}$, Y. Oya ${ }^{c}$, Y. Hatano ${ }^{\text {d }}$, \\ T. Otsuka ${ }^{\mathrm{e}}$, M. Oyaidzu ${ }^{\mathrm{f}}$, H. Kurotaki ${ }^{\mathrm{f}}$, T. Suzuki ${ }^{\mathrm{f}}$, D. Hamaguchi ${ }^{\mathrm{f}}$, K. Isobe ${ }^{\mathrm{f}}$, \\ N. Asakura ${ }^{\mathrm{f}}$, A. Widdowson ${ }^{\mathrm{g}}, \mathrm{K}$. Heinola ${ }^{\mathrm{h}}, \mathrm{M}$. Rubel ${ }^{\mathrm{i}}$ and JET Contributors $* *$ \\ ${ }^{a}$ National Institute for Fusion Science, Oroshi, Toki, Gifu 509-5292, Japan \\ ${ }^{b}$ Shimane University, Matsue, Shimane 690-8504, Japan \\ ${ }^{c}$ Shizuoka University, Shizuoka 422-8529, Japan \\ ${ }^{d}$ University of Toyama, Toyama 930-8555, Japan \\ ${ }^{e}$ Kindai University, Higashi-Osaka, Osaka, 577-8502, Japan \\ ${ }^{f}$ QST, Rokkasho Aomori 039-3212, Japan \\ ${ }^{g}$ EUROfusion Consortium, JET, Culham Science Centre, Abingdon, OX14 3DB, UK \\ ${ }^{h}$ University of Helsinki, PO Box 64, FI-00560 Helsinki, Finland \\ ${ }^{i}$ Royal Institute of Technology (KTH), 10044 Stockholm, Sweden \\ *Corresponding author: tokitani.masayuki@LHD.nifs.ac.jp
}

\begin{abstract}
Micro/nanoscopic observations on the surface of the divertor tiles used in the first campaign (20112012) of the JET tokamak with ITER-like Wall (JET ILW) have been carried out by means of several material analysis techniques. Previous results from the inner divertor were reported for a single poloidal section of the tile numbers 1, 3 and 4, i.e., upper, vertical and horizontal targets, respectively. The formation of the thick stratified mixed-material deposition layer on tiles 1 and 4, and erosion on tile 3 were identified. This study is mostly focused on the outer divertor: tiles 6, 7 and 8. In contrast to the inner tile, remarkable surface modifications have not been observed on the vertical target (tiles 7 and 8) where sputtering erosion and impurity deposition would have been almost balanced. Only a specific part of tile 6 (horizontal target) located near the exhaust channel was covered with a stratified ("geological-like”) mixed-material deposition layer which mainly included Be and $\mathrm{Ni}$ with the thickness of $\sim 2 \mu \mathrm{m}$. Special feature of this mixed layer was that a certain amount of nitrogen $(\mathrm{N})$ was clearly detected in the layer. Since the concentration of $\mathrm{N}$ varied with the depth position, it could be depended on the amount of that gas puffed for plasma edge cooling during the JET experimental campaign. In addition to the outer divertor tiles, a very interesting feature of the local erosion and deposition effects is reported in this paper.
\end{abstract}

Keywords: JET-ILW, divertor, erosion-deposition, fuel inventory

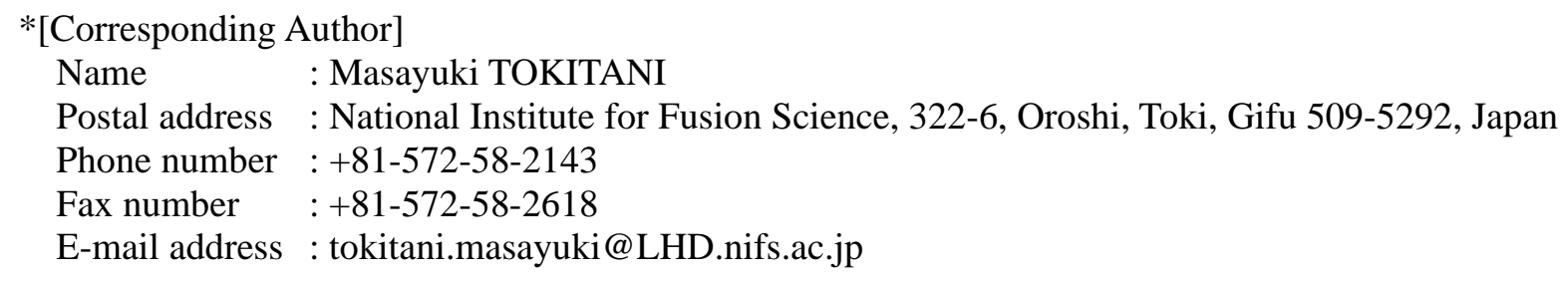

** See the author list of X. Litaudon et al., Nucl. Fusion 57 (2017) 102001. 


\section{Introduction}

In the D-T burning fusion reactors, tritium inventory on the plasma facing components (PFCs) should be kept to minimum not only for maintaining a fuel balance but also for ensuring radiological safety. Tungsten (W) is one of the potential candidates for PFCs in the fusion reactor because it has a low solubility of hydrogen isotopes and excellent thermal properties. ASDEX Upgrade has already demonstrated that deuterium inventory was significantly decreased under the full $\mathrm{W}$ wall compared with a carbon dominated wall [1]. In the initial material selection of the ITER PFCs, a combination of the carbon fiber composite (CFC) strike point tiles, W divertor and Be first wall had been considered [2]. However, the initial selection was changed to a new combination, the full $\mathrm{W}$ divertor and the beryllium (Be) first wall [3]. This new combination is predicted for strong reduction of tritium build-up compared to the initial material choice expected to be used in ITER PFCs [4].

The integrated tests of the ITER PFCs, the $\mathrm{W}$ divertor and Be first wall, in the large sized tokamak JET, the JET ITER-like wall (ILW) experiment [5], were started in the first ILW campaign from 2011 to 2012 [6]. Main objectives of the JET ILW are demonstrating an ability to operate a large high power tokamak and investigation of the material erosion, deposition, and fuel retention [7,8]. From the viewpoint of plasma wall interaction (PWI), many kinds of postmortem analysis for the first wall bulk/coated Be and the divertor W-coated CFC tiles used from 2011 to 2012 have been conducted by means of, e.g., ion beam analysis (IBA), thermal desorption spectroscopy (TDS) and secondary ion mass spectrometry (SIMS) by the European (EU) groups $[1,9,10,11,12,13,14]$. The analysis results revealed that the co-deposition layer with the impurity elements of $\mathrm{Be}, \mathrm{C}, \mathrm{O}$ and $\mathrm{W}$ plays a major role as a trapping site for the 
deuterium (D) $[10,11]$. More than one-half of the total amount of the retained D were retained in the divertor region, and the highest retention region corresponded to the thick deposition zone [10]. Based on the analysis results from the EU groups, a collaborative analysis program between JET groups and Japanese groups has been continued since 2014. A single poloidal section of the JET ILW divertor tiles samples were transferred to the International Fusion Energy Research Centre (IFERC), QST Rokkasho, Japan. Subsequent material analyses were carried out by several groups of domestic scientists. Previous studies have shown, that the apron of tile 1 is the main deposition part of the eroded Be from the first wall [13], and the highest amount of the D retention among all of the inner tiles was detected on this tile [10]. One of the noteworthy results from the Japanese group is the micro/nanoscopic observation of the subsurface region of the samples from the inner divertor tiles 1, 3 and 4, i.e., upper (apron), vertical and horizontal targets, respectively [15]. The formation of the thick stratified mixed-material deposition layer, composed of $\mathrm{Be}, \mathrm{W}, \mathrm{Ni}, \mathrm{O}$ and $\mathrm{C}$ on tiles 1 and 4 , and erosion on tile 3 were identified. As reported in earlier works, the mixed-material deposition layer on the apron of tile 1 included high amount of Be impurity. The transmission electron microscope (TEM) studies in Ref. [15] identified a dense nano-size bubble-like structure with size of over $100 \mathrm{~nm}$ in that Be rich layer. Judging from the size and density of the bubbles, formation of the bubble-like structure seems to be related to $\mathrm{D}$ retention in the layer. The observed micro/nano-structure clearly reflects the retention characteristics of the hydrogen isotopes on the tile. On the other hand, a large-size bubble formation in the deposition layer would cause undesired dust formation due to the exfoliation of the deposition layer itself. If the bubble-like structures are further growths or certain thermal stress is induced on the layer, a specific layer containing dense bubble-like structures might be fractured, and finally the entire layer will be exfoliated 
and act as a dust in the vacuum vessel [16]. However, to date only minuscular amounts of loose deposit material in the divertor has been observed on the JET ILW. From the fact mentioned above, it should be emphasized that information from the micro/nanoscopic observation is very important for assessment of both fuel inventory and behavior of dust.

In this study, therefore, additional analyses from the viewpoint of the micro/nanoscopic analysis were performed on mainly the outer divertor tiles 6, 7 and 8 . In addition, another sample from inner divertor tile 3, where the divertor strike point was often located, i.e., the erosion dominant tile, was also analyzed for comparison with outer divertor tiles.

\section{Experimental procedures}

Fig. 1 shows the poloidal cross-section of the JET ILW divertor with three representative plasma geometries and positions of all extracted surface samples with size of $17 \mathrm{~mm}$ in diameter and $5 \mathrm{~mm}$ in thickness transferred to International Fusion Energy Research Centre (IFERC), QST, Rokkasho, Japan [15,17]. In this study, samples of 8-10b, 7-1a and 6-6b from the outer divertor tiles of upper, vertical and horizontal, and samples of 3-6a from the inner vertical divertor tiles were selected for analysis. Photographs of those samples are also shown in the left and right row in Fig. 1. As explained in our previous work of Ref. [15], all JET divertor tiles except tile 5 consist of W coated CFC. Thickness of W-coating layer is 10-20 $\mu \mathrm{m}$ $[8,18]$. A set of special marker tiles composed by tiles 1, 4, 6, 7 and 8 were further coated with a W marker layer with a thickness of $\sim 3 \mu \mathrm{m}$ with a $\sim 3 \mu \mathrm{m}$ thick Mo interlayer between $\mathrm{W}$ marker layer and the thick $\mathrm{W}$-coating $[8,19]$. Tile 3 was without the $\mathrm{W}$ marker layer, i.e., the 
top surface was covered with Mo layer [8]. As with the previous work [15], prior to micro/nanoscopic analysis, $17 \mathrm{~mm} \phi$ disc samples were cut to small pieces by low speed cutter. Red lines and Red small symbols drawn on the sample photographs in Fig. 1 correspond to the cutting tracks and analyzed area in this study, respectively. After the cutting, micro/nanoscopic characterization of the tile surfaces were conducted by means of scanning electron microscope (SEM), scanning ion microscope (SIM), energy dispersive X-ray spectroscopy (EDS), electron probe micro analyzer (EPMA), focused ion beam (FIB) and TEM.

\section{Results and Discussions}

\subsection{Outer side tiles: tile 8 (8-10b), 7 (7-1a) and 6 (6-6b)}

Fig. 2 shows the surface and cross-sectional SEM images of 8-10b, 7-1a and 6-6b samples. The left side of each cross-sectional image corresponds to the top side (plasma facing side) of the samples. The W protection layers were artificially created on the top surface of each cross-sectional sample before FIB fabrication for protecting the top surface of the samples against the Ga fabrication ion beam in the FIB device.

In contrast to the previous analysis results from inner tiles [15], the 8-10b and 7-1a samples do not show remarkable deposition and erosion where sputtering erosion and impurity deposition would have been almost balanced. From the comparison with a virgin state of the tungsten coated graphite sample, sample surfaces of the 8-10b and 7-1a seem to be rather small net erosion. Previous work by means of the ion beam analysis (IBA) reported that smaller amounts of Be, C and D were observed on the surface of tiles 8 and 7 at all measured points [8]. 
Simultaneously, the double trapping peak of a D retention depth, i.e., at the surface and at the deeper region of the original $\mathrm{W}$-coating layer, was reported $[8,17,20]$.

On tile 6, prior studies reported that the thickness of the $\mathrm{W}$ layer increased homogeneously after exposure by about $500 \mathrm{~nm}$, while the Mo layer thickness did not show a notable change before and after exposure [8]. Since the adjacent bulk $\mathrm{W}$ tile 5 is a net tungsten erosion area, eroded $\mathrm{W}$ could be deposited on tile 6 [8]. On the other hand, a certain deposition of Be, C and D is observed at the central sloped part of tile 6, where the outer divertor strike point is often located. The 6-6b sample analyzed in this study corresponds to the central sloped part of the tile 6. Top view and cross-sectional surface microstructures on the 6-6b sample in Fig. 2 shows deposition feature and stratified deposition layer structure with the thickness of $\sim 2 \mu \mathrm{m}$ on the original $\mathrm{W}$-coating. Fig. 3 shows corresponding element depth profile of $\mathrm{W}, \mathrm{O}$, Mo Be, Ni and $\mathrm{N}$ detected by EPMA along with a dashed line in the cross-sectional SEM image of the 6-6b sample in Fig. 2. The selected X-ray lines for Ni and W are $\mathrm{K} \alpha$ and $\mathrm{M} \alpha$, respectively. Since the spatial resolution of the EPMA signal is expected to be $\sim 200 \mathrm{~nm}$, the intensity of each element at the interface between each layer and coating would not show a sharp drop or rise. The EPMA signal indicates that the deposition layer has a mixed-material feature included with many elements, and majority of the deposited elements were Be and $\mathrm{Ni}$. There are several sources of Ni. The main sources are: Inconel wall of the vacuum vessel, ICRH and lower hybrid antennae grills. Special feature of this mixed layer was that a certain amount of nitrogen $(\mathrm{N})$ was clearly detected in the layer. Since the concentration of $\mathrm{N}$ varied with the depth position, it is possible that it depended on the amount of that gas puffed for plasma edge cooling during the JET experimental campaign. For discussing the physical feature of this deposition layer, nano- 
scale observation by TEM was conducted. Fig. 4 shows the cross-sectional bright field (BF) TEM image of the mixed-material deposition layer of 6-6b sample. The deposition layer has a stratified, and a geological-like layer structure with the thickness of $\sim 2 \mu \mathrm{m}$. This layer structure must be caused by the plasma discharges and the operational history of JET. The dark image layers are mainly composed by relatively high-Z elements such as $\mathrm{W}$ and Mo, and the bright layers are mainly composed by low-Z elements such as Be, O, N and C. From the electron diffraction (ED) pattern image of the TEM observation indicates that the deposition layer has the amorphous-like structure. The depositional directivity does not show any sign on this deposition layer. Although the outer divertor strike points often located on this sample positon, nanostructure of the mixed-material deposition layer has very fine structure without large size cavities, e.g., nano-size bubble-like structures, confirmed in the 1-10c sample in the previous work [15]. Unfortunately, relevance between nanostructure of the mixed-material deposition layer and $\mathrm{N}$ depth profile cannot be identified by TEM image. The reported $\mathrm{D}$ retention on the position of the $6-6 \mathrm{~b}$ sample was estimated to be $\sim 2 \times 10^{22} \mathrm{D} / \mathrm{m}^{2}$ [8]. This value is almost the same as the horizontal target tile $4(4-10 \mathrm{~b})$ and vertical target tile $3(3-2 a)[8,15]$. The retention mechanism of the hydrogen isotopes in the mixed-material deposition layer could be due to the repetition of the impurity deposition and the hydrogen isotope injection. Thus, the sink against the hydrogen isotopes would have never been saturated as long as the deposition layer continues to be formed.

\subsection{Inner side strike point tile: tile 3 (3-6a)}

Original surface of the ILW has microscale surface roughness, e.g., micro-hills and - 
valleys. In the case of the high plasma influx regions with shallow incident angle of magnetic field lines, about $2-3^{\circ}$ to the surface parallel, such a roughness feature may lead to the local erosion and deposition effects in micrometer scale. Indeed, it was detected that both impurities, such as $\mathrm{Be}, \mathrm{Cr}, \mathrm{Fe}$ and $\mathrm{Ni}$ and fuel particle such as $\mathrm{D}$, have been locally accumulated on the microscale holes by means of ion beam analysis with micro beam [17]. This study focused on the inner side strike point tile 3 (3-6a), which original top surface is a Mo coating, to investigate a local erosion and deposition effects in micrometer scale.

Fig. 5-(a) and -(b) shows the secondary electron (SE) and the backscattered electron (BS) SEM images of 3-6a sample, respectively. Both images show exactly the same position. Generally, topological judgment is difficult by SE-SEM image, but BS-SEM image is helpful for judging. The local erosion and deposition process are affected by the shallow impact angle of the plasma, and by the target surface topography. In Fig. 5, many obvious micro-hill and micro-valleys can be found on the surface. The specific positions of micro-hill, micro-slope and micro-valley were marked by the cross markers as No. 1, 2 and 3, respectively, on both SEM images. The EDS point analysis spectra corresponding the cross markers of No. 1, 2 and 3 are shown in Fig. 5-(c). The hill position of No. 1 showed clear Mo peak with very small amount of $\mathrm{C}, \mathrm{O}$ and $\mathrm{W}$. That means there is an almost no deposition or net erosion region. Despite being only $10 \mu \mathrm{m}$ away from No. 1 position, Mo peak is dramatically decreased on the slope position of No. 2. Instead of Mo, very clear W peak, and obvious C, N, O and Ni peaks appeared. This indicates that erosion dominant feature is suddenly switched to deposition dominant feature even in the microscale distance. Since intensity of the deposited element peaks of W, C, N, O and $\mathrm{Ni}$ increased in the valley position of No. 3, the tendency of the deposition dominant feature becomes stronger towards the valley position. 
For more precise analysis of the local erosion and deposition effects in micrometer scale, cross-sectional SEM observation and EPMA analysis were conducted. Fig. 6-(a) shows the SIM image of the 3-6a sample. This image is not in the exact same position with Fig. 5, but is almost the same area and geometry. A cross-sectional SEM image extracted from the red rectangle position is marked in Fig. 5-(a) by means of FIB technique shown in Fig. 5-(b). The W protection layer was artificially created on the top surface of the sample against the Ga fabrication ion beam in the FIB device. The hill, slope and valley as mentioned in Fig. 5 can be clearly identified in this cross-sectional image. It is clear that the hill position cannot see any net deposition due to plasma interactions. On the other hand, thickness of the deposition layer is gradually increased from the slope to the valley positions. The deposition layer on the valley position has complex multilayer structure. For confirming the element distribution in the deposition layer, the EPMA analysis was conducted in the same cross-sectional sample of Fig. 6-(b). Fig. 6-(c) shows the EPMA element mapping of the deposited elements of Mo, W, Be, C, $\mathrm{N}$ and Ni. Although the EDS analysis of Fig. 5 was not able to identify the Be deposition, it is confirmed that the majority of the deposited element is Be, and certain amounts of $\mathrm{W}, \mathrm{C}, \mathrm{N}$, and $\mathrm{Ni}$ are also deposited together with Be. Judging from the deposition feature of both Figs. 5 and 6, incoming direction of the impurities and plasma particles flow could be from the left hand side to the right hand side of the SEM images. This direction qualitatively agrees with the magnetic field line direction of the JET tokamak. Such local erosion deposition phenomena would be caused by a shallow connection angle of the magnetic field line to the tile surface. The results as shown in Figs. 5 and 6 are very interesting because both erosion and deposition simultaneously occurred in the very local area around the $\sim 10 \mu \mathrm{m}$ square. Due to the shallow incident angle of the impurities and plasma particles, the hill position would enhance the erosion 
by plasma influx. On the other hand, since the slope and valley positions were located on a shadow area of the hill, sputtering erosion could be suppressed. In contrast, impurity deposition was enhanced.

This result indicates that local erosion and deposition effects in micrometer scale would lead to an inhomogeneous retention of the hydrogen isotopes on the erosion dominant surface e.g., the strike point of the divertor plasma.

\section{Summary}

Micro/nanoscopic observations on the surface of the divertor tiles used in the first campaign (2011-2012) of JET ILW have been carried out by means of several material analysis techniques.

In contrast to the inner tiles results [15], remarkable surface modifications have not been detected on the vertical target (tile 7 and 8) where sputtering erosion and impurity deposition would have been almost balanced. Only a specific part of tile 6 (6-6b) located near the exhaust channel was covered with stratified (“geological-like”) mixed-material deposition layer which mainly included Be and Ni with the thickness of $\sim 2 \mu \mathrm{m}$. A special feature of this mixed layer was that a certain amount of nitrogen $(\mathrm{N})$ was clearly detected in the layer. Since the concentration of $\mathrm{N}$ varied with the depth position, it could have depended on the amount of that gas puffed for plasma edge cooling during the JET experimental campaign. Since a certain amount of $\mathrm{D}$ was retained on the same position of 6-6b [8], the mixed-material deposition layer could act as the sink for hydrogen isotopes. In general, if a mixed-material deposition layer is exfoliated, it could behave as the tritium content dust in the vacuum vessel. A very interesting feature of the local erosion and deposition effects in micrometer scale has been found on the 
inner divertor tile sample of 3-6a. Due to the shallow incident angle of the impurities and plasma particles on the original roughness surface structure such as micro-hills and valleys, enhancement of the sputtering erosion and impurity deposition simultaneously occurred in a very small area around the $\sim 10 \mu \mathrm{m}$ square. The local erosion and deposition effects in micrometer scale would lead to an inhomogeneous retention of the hydrogen isotopes on the erosion dominant surface, e.g., the strike point of the divertor plasma.

The results obtained in this study revealed that information regarding the micro/nanoscopic surface modification is very important for predicting the lifetime of the plasma facing components, tritium inventory, and dust formation. This micro/nanoscopic observation technique will continue to be applied to future JET ILW tile analysis.

\section{Acknowledgments}

This work has been carried out within the framework of the EUROfusion Consortium and has received funding from the Euratom research and training program 2014-2018 under grant agreement No 633053. The views and opinions expressed herein do not necessarily reflect those of the European Commission. This work has been supported by the ITER Broader Approach Activity. Dr. Jari Likonen and members of his team are gratefully acknowledged for the preparation of samples from the divertor tiles. 


\section{References}

[1] M. Mayer et al., J. Nucl. Mater. 390-391 (2009) 538-543.

[2] G. Federici et al., Plasma Phys. Control. Fusion 45 (2003) 1523-1547.

[3] R.A. Pitts et al., J. Nucl. Mater. 438 (2013) S48-S56.

[4] J. Roth et al., Plasma Phys. Control. Fusion 50 (2008) 103001.

[5] G.F. Matthews et al., Phys. Scr. T128 (2007) 137-143.

[6] J. Likonen et al., Phys. Scr. T159 (2014) 014016.

[7] G.F. Matthews et al., Phys. Scr. T145 (2011) 014001.

[8] M. Mayer et al., Phys. Scr. T167 (2016) 014051.

[9] S. Krat et al., J. Nucl. Mater. 456 (2015) 106-110.

[10] K. Heinola et al., Phys. Scr. T167 (2016) 014075.

[11] K. Heinola, et al., J. Nucl. Mater. 463 (2015) 961-965.

[12] S. Brezinsek, JET-EFDA contributors J. Nucl. Mater. 463 (2015) 11-21.

[13] S. Brezinsek et al., Nucl. Fusion 55 (2015) 063021.

[14] J. Likonen, et al., Phys. Scr. T167 (2016) 014074.

[15] M. Tokitani et al., Fusion Eng. Des. 116 (2017) 1-4.

[16] S. Masuzaki et al., Phys. Scr. T170 (2017) 014031.

[17] H. Bergsåker et al., J. Nucl. Mater. 463 (2015) 956-960.

[18] C. Ruset et al., Fusion Eng. Des. 84 (2009) 1662-1665.

[19] M. Rubel et al., J. Nucl. Mater. 438 (2013) S1204-S1207.

[20] H. Bergsåker et al., Phys. Scr. T167 (2016) 014061. 


\section{Figure captions}

Fig. 1 Poloidal cross-section of the JET ILW divertor with three representative plasma geometries [15,17], and positions of samples. Photographs of four analyzed samples in this study are shown in the left and the right rows. Lines and symbols on the samples are the cutting tracks and the analyzed positions, respectively.

Fig. 2 Surface; upper series, and cross-sectional; lower series SEM images of 8-10b, 7-1a and 6-6b samples.

Fig. 3 Element depth profile of W, O, Mo Be, Ni and N detected by EPMA along with a horizontal dashed line in the cross-sectional SEM image of the 6-6b sample in Fig. 2

Fig. 4 Cross-sectional BF-TEM image of 6-6b sample.

Fig. 5 Secondary electron (SE) (a) and backscattered electron (BS) (b) SEM images of same area of the 3-6a sample and corresponding EDS point analysis spectra (c) on 1, 2 and 3 positions on the SEM images. Direction of the magnetic field line (B direction) on the samples roughly indicated by an arrow on the SEM images.

Fig. 6 (a) SIM image of the surface of the 3-6a sample. (b) A corresponding cross- 
sectional SEM image extracted from the red rectangle area in the SIM image of (a). (c) EPMA mappings. Direction of the magnetic field line (B direction) on the samples roughly indicated by an arrow. 
Fig. 1 One column wide

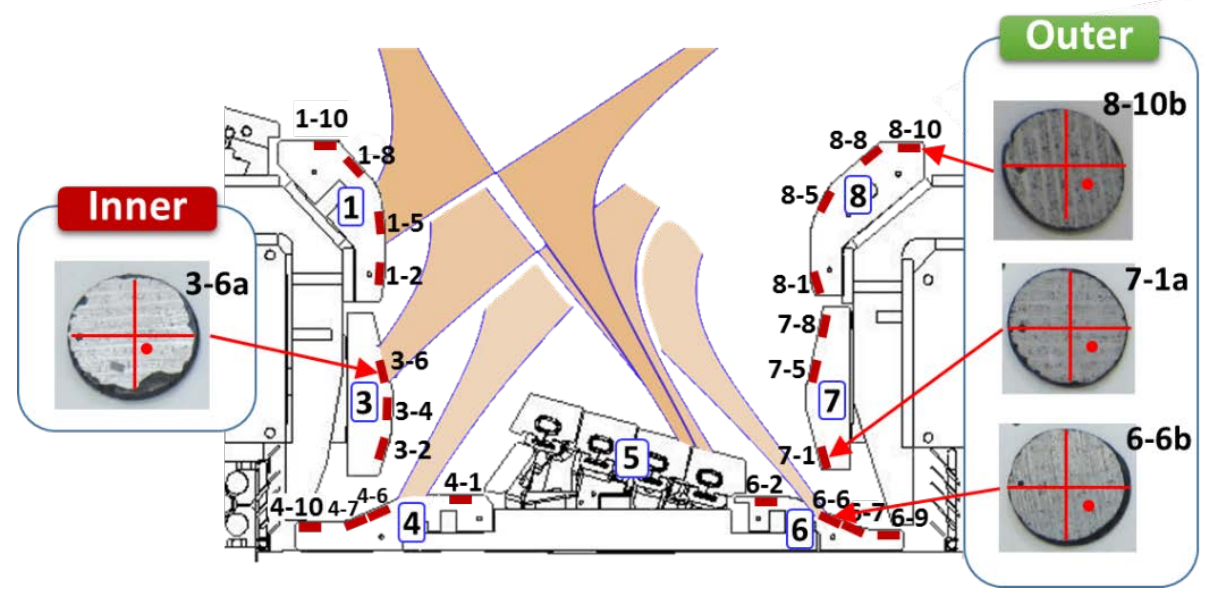


Fig. 2 Two columns wide

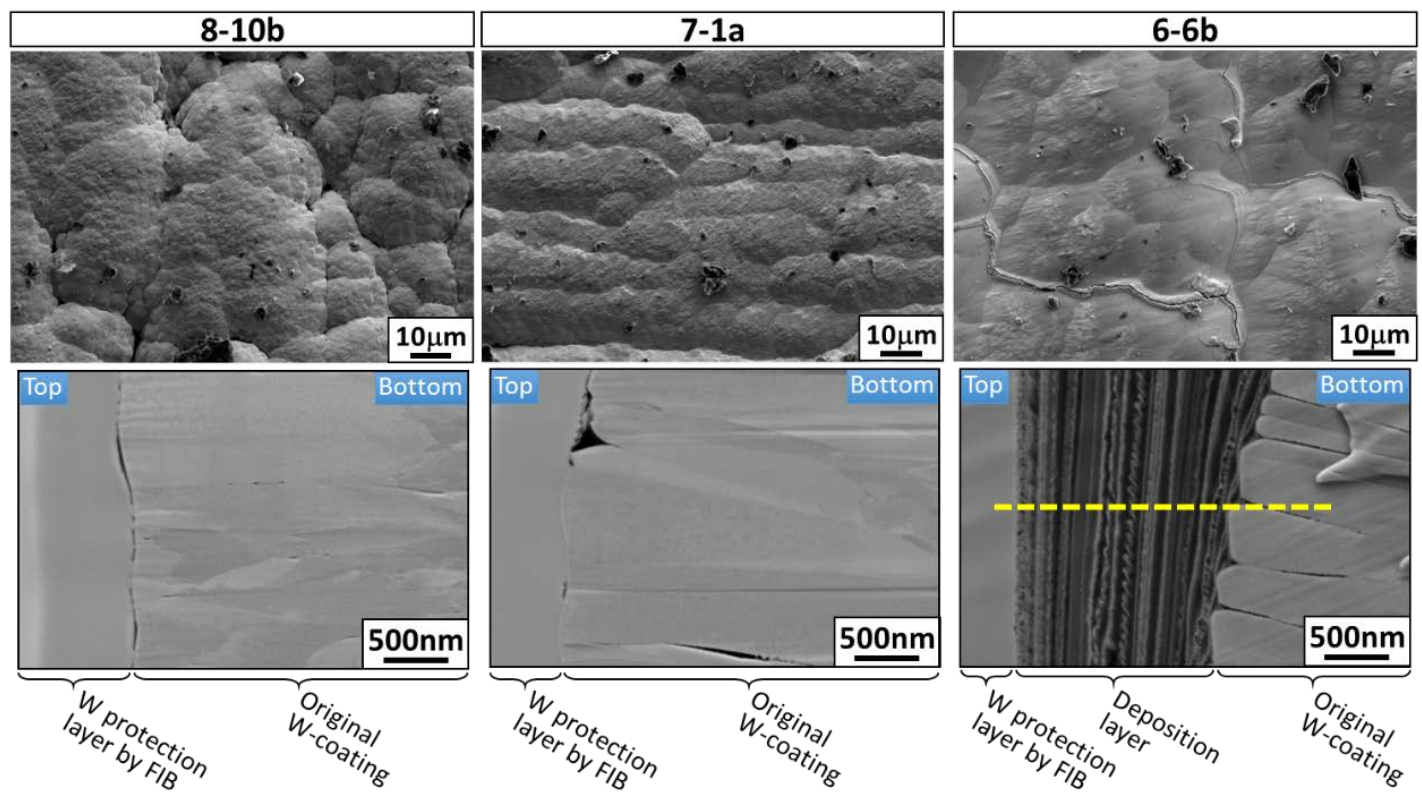


Fig. 3 One column wide

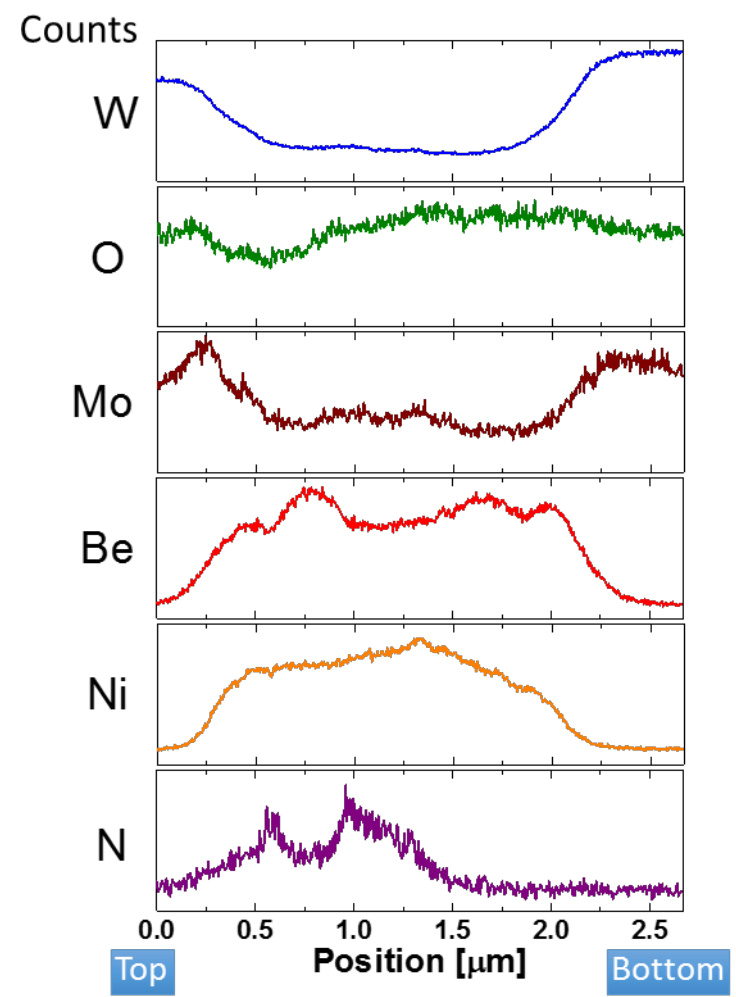


Fig. 4 Two columns wide

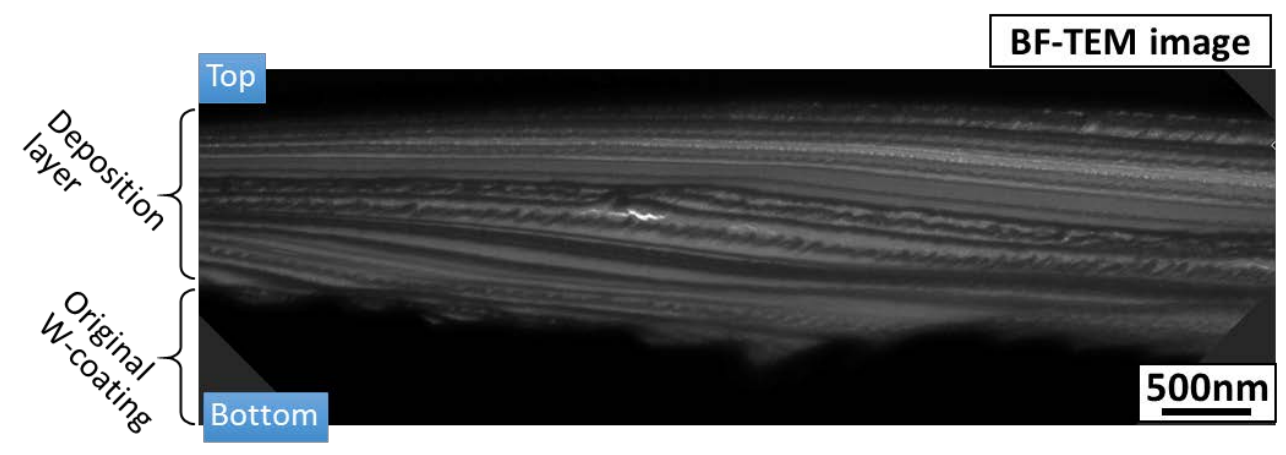


Fig. 5 Two columns wide
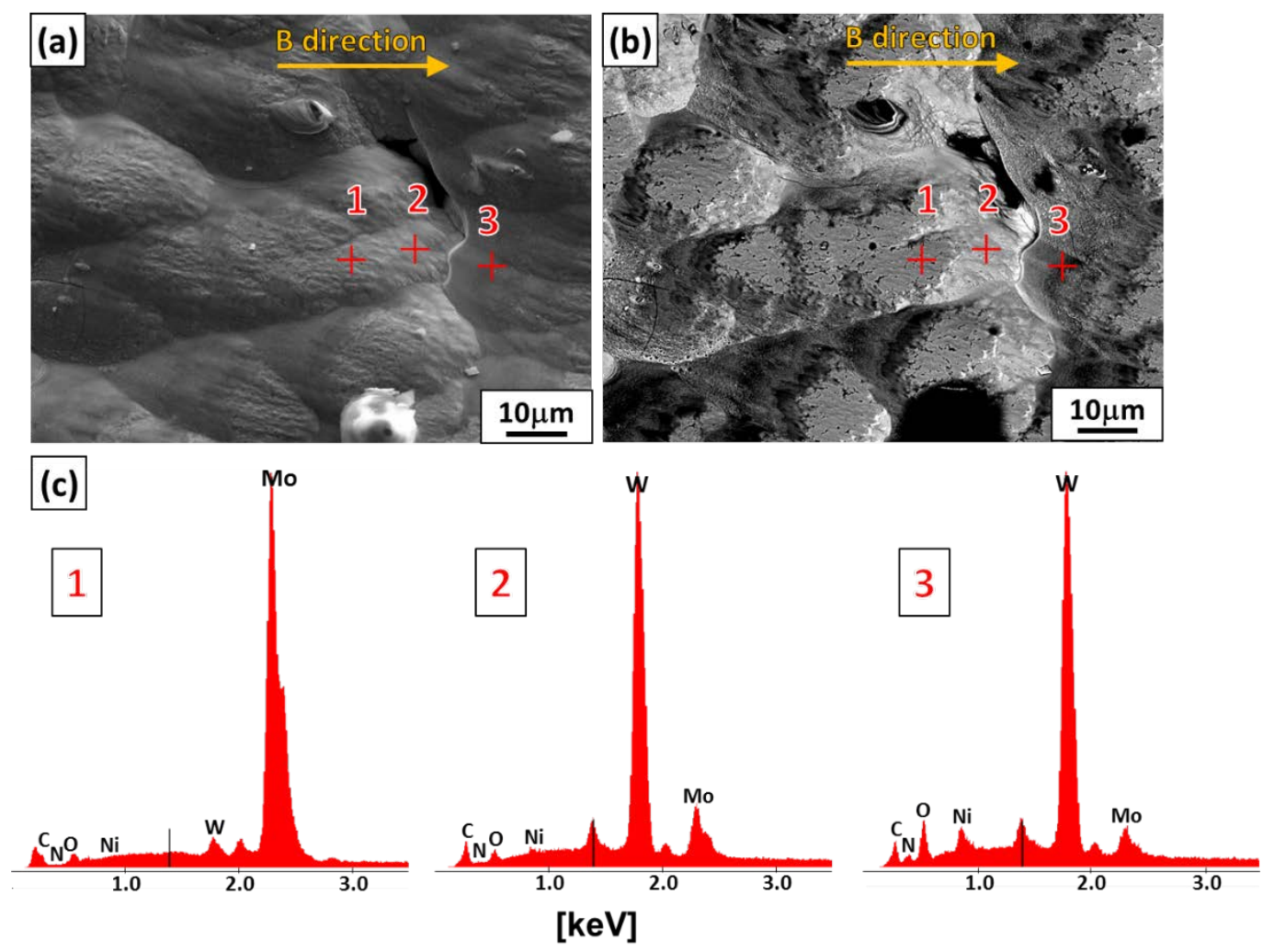
Fig. 6 One column wide
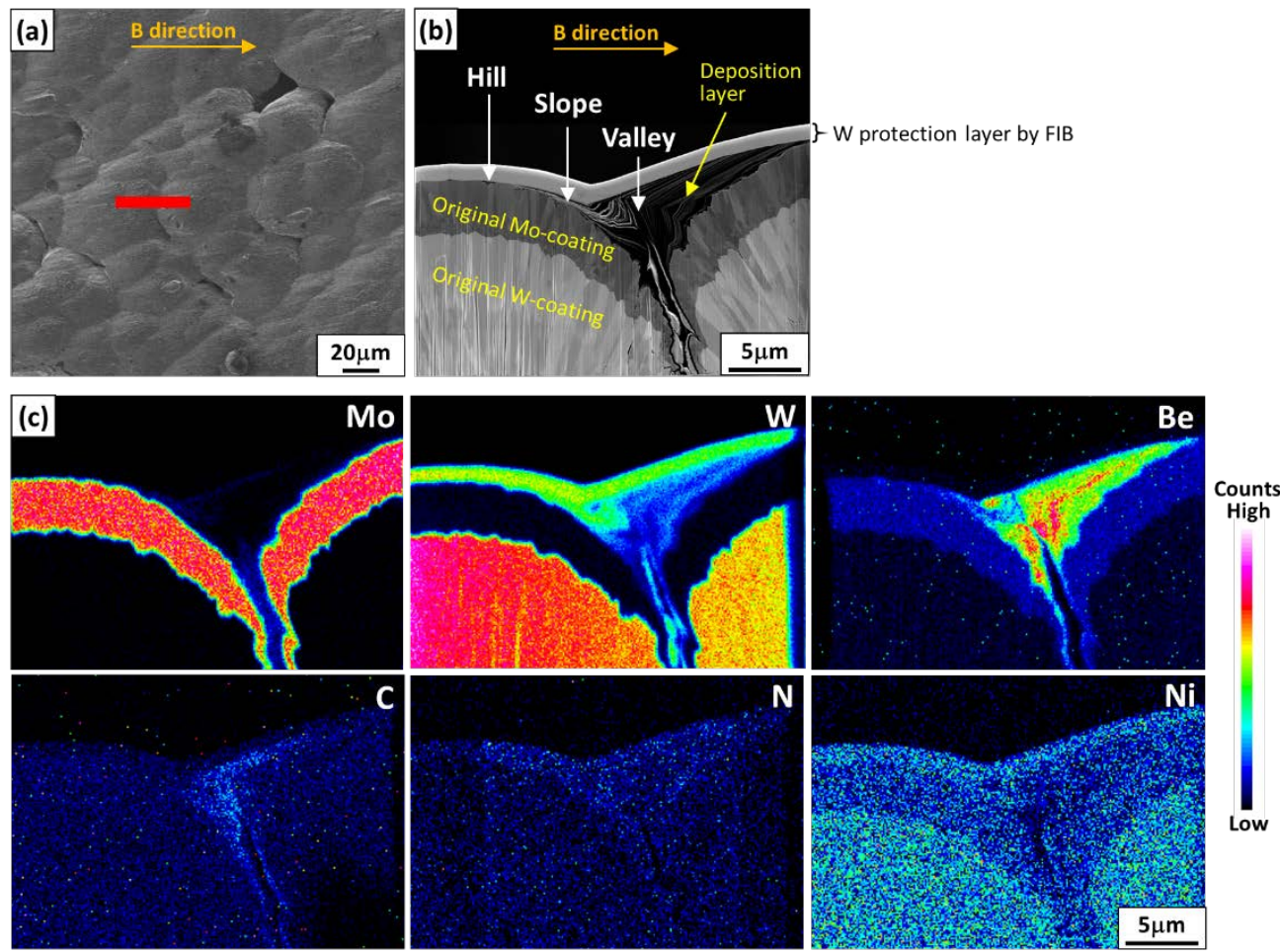